\title{
Hutchinson Gilford Progeria Syndrome: A Therapeutic Approach via Adenoviral Delivery of CRISPR/cas Genome Editing System
}

\author{
Walter Arancio*, Swonild Ilenia Genovese, Giuseppe Pizzolanti and Carla Giordano
}

Section of Cardio-Respiratory and Endocrine-Metabolic Diseases, Biomedical Department of Internal and Specialist Medicine (Di.Bi.M.I.S.), University of Palermo, Piazza delle Cliniche 2, 90127 Palermo, Italy

\begin{abstract}
Hutchinson-Gilford Progeria Syndrome (HGPS) is a rare human genetic disease caused by mutations in the LMNA gene. LMNA codes for structural components of the nuclear lamina. Alterations of nuclear lamina lead to a very variable class of diseases known as laminopathies. In detail, HGPS manifests a severe premature ageing phenotype due to the accumulation of a dominant negative form of lamin-A called progerin. With current treatments, the life expectancy of HGPS patients does not exceed their second decade. Death is usually due to cardiovascular complications.

Recently, a new technology for mammals in vivo gene editing has been developed: the clustered regularly interspaced short palindromic repeats/Cas protein (CRISPR/Cas) system. The CRISPR/Cas technology permits to edit the genome at specific loci. Even if the CRSIPR/Cas constructs are transiently administered to the target cells, the genome editing is permanent. The advantages of the combination of non-integrating transient vectors in combination with the CRISPR/Cas constructs could give rise to a secure approach for the treatment of disease of genetic origin, especially those caused by dominant negative mutations, such as HGPS.
\end{abstract}

A potential application of non-integrating transient vectors carrying CRISPR/Cas constructs for the treatment of HGPS will be discussed in detail.

Keywords: HGPS; Hutchinson-Gilford Progeria Syndrome; Progeria; LMNA; Lamin-A; Progerin; CRISPR; CRISPR/Cas; CRISPR/ Cas9; Cas9; Gene therapy; Adenovirus; Adenoviral vectors

\section{Hutchinson Gilford Progeria Syndrome}

Progeroid syndromes are monogenic systemic diseases where affected individuals show phenotypes and clinical conditions associated with ageing at a younger age than expected.

Hutchinson-Gilford Progeria Syndrome (HGPS) is a very rare disease, reported to occur in 1 in 8 million newborns. Less than 150 cases have been reported in the scientific literature since the condition was first described [1]. At cellular and molecular level, HGPS is characterized by severe alterations of the nuclear architecture, chromatin organization, epigenetics and regulation of transcription. Interestingly, the changes observed in HGPS are often similar to those observed in the very old $[1,2]$.

HGPS children typically look normal at birth and in early infancy, but then they "fail to thrive". They develop a typical facial appearance with complete alopecia, aged-looking skin together with joint abnormalities and lipodistrophy. The intellectual and motor skill developments are normal.

A severe arteriosclerosis usually begins since childhood. The risk of having a heart attack or stroke is high and the condition usually worsens over time. HGPS children typically die of heart disease at an average age of 13 years $[1,2]$.

HGPS is caused by a de novo autosomal dominant synonymous G608G (nucleotide C1824T) mutation within exon 11 of the LMNA gene. This mutation activates a cryptic splicing site that leads to the production of progerin protein instead of canonical lamin-A. Progerin differs from lamin-A because it lacks 50 aminoacids and because it becomes constitutively farnesylated and anchored into the inner nuclear membrane [3]. In detail, during the canonical maturation of prelamin-A, the CaaX motif on its $\mathrm{C}$-terminus is farnesylated on cysteine, the last 3 amino acids are lost, and pre-lamin- $A$ is anchored into the inner nuclear membrane. In a second time, the metallopeptidase Zmpste24 cleaves the last 18 amino acids to produce the mature, unfarnesylated lamin-A. In progerin, the Zmpste24 cleavage site is lost, so progerin tail cannot be removed, it remains constitutively farnesylated and anchored into the inner nuclear membrane, and it becomes the causative agent of HGPS [1-4]. In fact, a mouse model where the activity of mouse orthologue of Zmpste24 is impaired is currently used as model of HGPS and actually this mouse model recapitulates many aspects of human HGPS [5].

Within the nucleus, lamins can directly and indirectly bind to chromatin. Lamin-associated regions tend to be heterochromatic and transcriptionally silent [4]. In fact, HGPS causes severe global epigenetic alterations that resemble those that occur in elderly specimens. Histonecovalent modifications, histone variants, DNA methylation, chromatin remodelers, chromatin architecture and miRNAs are all altered in HGPS [6-8]. Interestingly, the alteration of the nuclear architecture is so severe that it can be cytologically seen by the typical nuclear lobulation or blebbing [2,3]. Moreover, in HGPS mitochondrial dysfunction [9], altered cell-cycle regulation [10], impaired DNA repair mechanisms

${ }^{*}$ Corresponding author: Walter Arancio, Section of Cardio-Respiratory and Endocrine-Metabolic Diseases, Biomedical Department of Internal and Specialist Medicine (Di.Bi.M.I.S.), University of Palermo, Piazza delle Cliniche 2, 90127 Palermo, Italy, Tel: +390916552138; E-mail: walter.arancio@unipa.it

Received October 10, 2014; Accepted December 22, 2014; Published December 29, 2014

Citation: Arancio W, Genovese SI, Pizzolanti G, Giordano C (2014) Hutchinson Gilford Progeria Syndrome: A Therapeutic Approach via Adenoviral Delivery of CRISPR/cas Genome Editing System. J Genet Syndr Gene Ther 6: 256. doi:10.4172/2157-7412.1000256

Copyright: (c) 2014 Arancio W, et al. This is an open-access article distributed under the terms of the Creative Commons Attribution License, which permits unrestricted use, distribution, and reproduction in any medium, provided the original author and source are credited. 
[1,4], telomere dysfunctions [11], and quicker cellular senescence [11] have been reported $[8,11]$.

The major difference between HGPS and physiological ageing is the occurrence of cancer; in fact, in HGPS patients, cancer transformation is a very rare event [12].

\section{Current Therapies}

To date, no effective therapy is available for HGPS. The cardiovascular conditions are carefully monitored and the use of lowdose aspirin is a usually recommended medication [1].

The use of Farnesyl Transferase Inhibitors (FTIs), such as the lonafarnib, showed some limited improvement in the conditions of the patients [13]. Several pharmacological approaches are tried to ameliorate specific conditions [1] (e.g. the zoledronic acid used to increase bone mineral density).

Up to date, the only treatment that has the aim to revert the causative agent of HGPS is the FTIs treatment that tries to reduce the amount of farnesylated progerin. Unluckily, FTIs treatments showed only modest results [13].

In order to find an effective treatment for HGPS the possibility of using gene therapy is currently taken into account. The use of the CRISPR/Cas gene editing system delivered by non-integrating viral vectors might be an interesting approach for the genetic therapy of HGPS

\section{CRISPR/Cas System}

CRISPR/Cas system was discovered as acquired immune system in bacteria and archaea [14]. In bacteria, CRISPR/Cas system degrades non-self DNA via a RNA-guided DNA cleavage. 3 types of CRISPR-Cas systems have been isolated [15]. One of those, the type II CRISPR/Cas system has been transformed into a high efficient and sequence-specific genome-editing tool [16].

In bacteria, Type II CRISPR involves a CRISPR RNA (crRNA), a trans-activating CRISPR RNA (tracrRNA), and the Cas9 protein. Portions of the DNA of the invading agent, usually a virus, are inserted within the bacterial genomic CRISPR loci. Those sequences are transcribed into specific crRNA. crRNAs determine the target DNA sequence that will be manipulated by the CRISPR/Cas system. The universal tracrRNA together with the sequence specific crRNA guide the Cas9 double-stranded DNA endonuclease to cleave specific DNA sequences. Cas9 activity generates double strand breaks (DSBs) $[14,15]$.

During the efforts to use the CRISPR/Cas system in genetic engineering, the system has been simplified. Now, it is routinely used a chimeric guide RNA (gRNA) consisting of a fusion of crRNA and tracrRNA. A sequence specific gRNA can efficiently guide the Cas9 system to generate DSBs within the desired sequence with high efficiency and specificity [16-18].

The CRISPR/Cas system can be used in many ways. The two most used applications are the gene disruption creating either an indel (small insertions/deletions) within the gene of interest or sequence specific gene editing $[16,17]$. The changes introduced by these uses of the CRISPR/Cas system are permanent. Several modified CRISPR/Cas systems have been developed to increase the specificity of the DSBs to minimize the risk of off-target cuts [16-18].

\section{Indels}

The DSB is repaired usually by the Non-Homologous End Joining
(NHEJ) DNA repair pathway. During NHEJ repair, InDels may occur. One or two nucleotides indels alter the Open Reading Frame (ORF). That usually leads to an early truncation of the protein downstream of the DSB site [16-18]. It is worth to note that an early truncation near the C-ter of the progerin should lead to a protein that cannot be farnesylated that highly resemble a mature non-pathogenic wild type lamin-A.

\section{Gene editing}

Alternatively, the CRISPR/Cas system can also be used to specifically edit the target sequence. When a DNA sequence with a high degree of homology to the sequence immediately upstream and downstream of the DSB is present, the Homology Directed Repair (HDR) mechanism can be used instead of NHEJ repair. In the presence of a suitable template, HDR can faithfully introduce specific nucleotide changes at the DSB site. The donor DNA template can be transfected into the cell together with the gRNA/Cas9 or can be present within the genomic DNA [16-18]. It is worth to note that the wild type LMNA allele can be used as donor DNA in the case of a DSB that specifically occurs within the progerin-coding gene, even if the frequency of HDR correction might be too low in absence of a co-transfected donor template.

\section{Vector Selection for Gene Therapy}

Gene therapy aims to manipulate the genome as ultimate treatment for various disorders. Gene therapy applied to patients who suffer from genetic disorders aims to revert their phenotype to wild type [19]. Traditional gene therapy tried to introduce one transgene to compensate for a loss of function [19].

A critical step in this process is to find an efficient transduction technique to be used in human cells. Viral vectors have showed great potentiality for their use in gene therapy. Viral vectors have been engineered to be not-pathogenic defective viruses to increase their safety and capacity. They are now tailored to their specific applications and far away from their pathogenic wild type counterpart [19]. Viral vectors con be roughly divided into two subgroups: integrating and not-integrating viral vectors [19-21].

\section{Integrating vectors}

Lentiviral derived vectors represent a typical example of integrating viral vectors. These vectors have the capacity of integrating their retrotranscribed genome within the host genome with the advantage of a permanent and heritable change and transgene expression. The drawback of this system is that the viral genome is inserted at a random position and it can perturb the function of cellular genes and thus promote the development of cancer [20].

\section{Non integrating vectors}

A typical example of non-integrating vectors is represented by Adenoviral derived vectors. These vectors carry their genetic material in the form of double-stranded DNA that is not incorporated into the host genome. Adenoviral vectors oncogenicity is virtually non-existent, but they have the drawback to induce only transient expression of the transgenes within the host cells [21], and the drawback to have the ability to induce immune responses [21]. A promising class of non integrating vectors are represented by the Adeno-associated virus derived vectors, that cause a very mild immune response, possess a wide cell host spectrum but they have the drawback that rarely exert a random integration in the host genome [22,23]. Nevertheless, they showed good promise in therapy [24]. Engineered non-integrating lentiviral vectors share many promises and drawbacks of Adenoassociated virus derived vectors [25]. 


\section{Why Use Adenoviral Vectors Carrying CRISPR/Cas Constructs for the Treatment of HGPS}

The use of adenoviral-derived vectors carrying CRISPR/Cas constructs permits to overcome the limitation of classical gene therapy. This approach has given the first positive results [26-30] and, in authors' opinion, their numbers will rise quickly. The adenoviral derived vectors don't integrate within the host genome, so the possibilities that they can induce cancer transformation is remote [21]. Their major drawback is that they can only exert their functions transiently. The CRISPR/Cas system overcames this problem. In fact, the transiently derived CRISPR/Cas system can permanently modify the genome at the required loci [16]. Then, during the cell divisions, the adenoviral vectors and construct will be lost, but the genome modification will be retained. The combination of those two technologies transforms a transient expression of the CRISPR/Cas constructs into a permanent genome editing.

The use of this technology seems particularly suited for the treatment of genetic diseases caused by a dominant negative mutation, such in the case of HGPS. In detail, specific genome editing in the mutated locus can activate either NHEJ repair system [16-18], that will lead the production of a truncated form of progerin that highly resemble the wild type unfarnesylated mature lamin-A [1], or the HDR repair system [16-18] using either the wild type allele as donor template or a co-transfected donor plasmid, that will lead to the production of wild type lamin-A. Whatever it will be the case, the change will be permanent, and it will be inherited by the daughter cells through the entire lineage. The first evidences of the adenoviral-based CRISPR/ Cas treatment in mammals are very promising [26,27], and they seem to assure a high efficiency of genome editing with very few off-target events.

It is worth to note that an adenoviral approach to treat HGPS has been previously investigated. Induced pluripotent stem cells derived from patients have been engineered via adenoviral vectors to induce a homologous recombination-based gene correction [31]. Even if the approach described there does not make use of CRISPR/Cas technology, it gives the proof of principle that adenoviral vectors are a feasible tool to treat HGPS. Moreover, the authors showed that the same approach might be used to engineer mesenchymal stem cells (MSCs), addressing the major concern to define the ideal cellular targets for genetic correction. In fact, at least two different strategies might be exploited. In HGPS patients, given the hereditary genetic origin of the syndrome, all cells of the affected individuals are involved, with the notable exception of the nervous system thanks probably to an epigenetic phenomenon [8,32]. By this perspective, targeting and correcting progenitor stem cells might hopefully repopulate differentiating cell lineages with corrected cells thus leading to the development of healthy tissues. Even if that could be an ideal strategy, it is difficult to be realized in vivo. An ex vivo approach using autologous MSCs reprogramming and transplantation might be a good compromise; in fact MSCs are easy to collect [33], MSCs derived tissues are specifically damaged in HGPS [1], the stem cells homing effect might help to colonize and populate damaged tissues [34] and this approach has given promising results in the past $[31,35]$.

As mentioned before, heart disease is the major cause of death in HGPS patients. A second strategy worth to exploit is to specifically target cardiac and blood vessels for correction. Interestingly, an engineered adenoviral vector that enables multi-organ vascular endothelial targeting in vivo has been recently reported [36].
It is worth to note that the cells with their genome edited to resemble the wild type condition, might compete with the cells that retain the HGPS mutation, and by competition and selection they might gain a selective advantage in colonizing the different body part [37], magnifying in vivo the effect of the treatment.

\section{Conclusions}

HGPS is a rare condition caused by a dominant negative mutation in the LMNA gene that leads to the accumulation of a mutated form of the nuclear lamin-A called progerin. Recent innovations in genetic engineering techniques have lead to the production and refining of the CRISPR/Cas tool for genomic editing. This tool shows great potentiality for its use in gene therapy. In fact, it permits to manipulate the site of pathogenic mutations using transient viral vectors such as the adenoviral-derived vectors or other non-integrating vectors. The great advantage is that even if the vector cannot integrate within the host, the constructs can permanently manipulate the genome, adding to the safety of the vector, the possibility to introduce permanent and sitespecific genomic modifications.

Specifically, due to the peculiar nature of the HGPS causative negative dominant mutation within the $\mathrm{C}$-terminus coding region, this approach might lead to the reversion to a wild type phenotype independently from the repair mechanism activated by the introduction of the DSB, either NHEJ or HDR. It is worth to note that the same approach might be taken into account for the treatment of other genetic diseases caused by dominant negative mutations, for which the delivery of specifically tailored CRISPR/Cas constructs by non-integrating vectors might prove to be an efficient therapeutic strategy.

\section{Acknowledgement}

Our thanks go to Giordano Bruno, Nikola Tesla and Barbara McClintock fo their inspiring vision.

\section{Funding}

This work has been partially funded by PON01_00829 and RIMEDR (Rete Regionale Integrata Clinico-Biologica per Medicina Rigenerativa) CUP G73F12000150004.

\section{References}

1. Gonzalez JM, Pla D, Perez-Sala D, Andres V (2011) A-type lamins and Hutchinson-Gilford progeria syndrome; pathogenesis and therapy. Front Biosci 3:1133-1146.

2. Liu GH, Barkho BZ, Ruiz S, Diep D, Qu J, et al. (2011) Recapitulation of premature ageing with iPSCs from Hutchinson-Gilford progeria syndrome. Nature 472:221-225

3. Dechat T, Pfleghaar K, Sengupta K, Shimi T, Shumaker DK, Solimando L, et al. (2008) Nuclear lamins: major factors in the structural organization and function of the nucleus and chromatin. Genes Dev 22:832-853.

4. Prokocimer M, Barkan R, Gruenbaum Y (2013) Hutchinson-Gilford progeria syndrome through the lens of transcription. Aging Cell 12:533-543.

5. Osorio FG, Obaya AJ, López-Otín C, Freije JM (2009) Accelerated ageing: from mechanism to therapy through animal models. Transgenic Res 18:7-15

6. Arancio W, Giordano C, Pizzolanti G (2013) A ceRNA analysis on LMNA gene focusing on the Hutchinson-Gilford progeria syndrome. J ClinBioinforma 3: 2.

7. Arancio W (2012) A bioinformatics analysis of Lamin-A regulatory network: a perspective on epigenetic involvement in Hutchinson-Gilford progeria syndrome. Rejuvenation Res 15:123-127.

8. Arancio W, Pizzolanti G, Genovese SI, Pitrone M, Giordano C (2014) Epigenetic involvement in Hutchinson-Gilford progeria syndrome: a minireview. Gerontology. 60:197-203

9. Rivera-Torres J, Acín-Perez R, Cabezas- Sánchez P, Osorio FG, GonzalezGómez C, et al. (2013) Identification of mitochondrial dysfunction in Hutchinson- 
Citation: Arancio W, Genovese SI, Pizzolanti G, Giordano C (2014) Hutchinson Gilford Progeria Syndrome: A Therapeutic Approach via Adenoviral Delivery of CRISPR/cas Genome Editing System. J Genet Syndr Gene Ther 6: 256. doi:10.4172/2157-7412.1000256

Gilford progeria syndrome through use of stable isotope labeling with amino acids in cell culture. J Proteomics 91:466-477.

10. Johnson BR, Nitta RT, Frock RL, Mounkes L, Barbie DA, et al. (2004): A-type lamins regulate retinoblastoma protein function by promoting subnuclear localization and preventing proteasomal degradation. ProcNatIAcadSci USA 101:9677-9682.

11. Ghosh S, Zhou Z (2014) Genetics of aging, progeria and lamin disorders CurrOpin Genet Dev 26C:41-46.

12. Fernandez P, Scaffidi P, Markert E, Lee JH, Rane S, et al (2014) Transformation Resistance in a Premature Aging Disorder Identifies a Tumor-Protective Function of BRD4. Cell Rep 9:248-260.

13. Ullrich NJ, Kieran MW, Miller DT, Gordon LB, Cho YJ, et al. (2013) Neurologic features of Hutchinson-Gilford progeria syndrome after lonafarnib treatment. Neurology 81:427-430.

14. Horvath P, Barrangou R (2010) CRISPR/Cas, the immune system of bacteria and archaea. Science 327: 167-170.

15. Carte J, Christopher RT, Smith JT, Olson S, Barrangou R et Al. (2014) The three major types of CRISPR-Cas systems function independently in CRISPR RNA biogenesis in Streptococcus thermophilus. MolMicrobiol 93:98-112.

16. Sampson TR, Weiss DS (2014) Exploiting CRISPR/Cas systems for biotechnology. Bioessays J 36:34-38.

17. Munoz IM, Szyniarowski P, Toth R, Rouse J, Lachaud C (2014) Improved Genome Editing in Human Cell Lines Using the CRISPR Method. PLoS One 9:e109752.

18. O'Connell MR, Oakes BL, Sternberg SH, East-Seletsky A, Kaplan M, et a (2014) Programmable RNA recognition and cleavage by CRISPR/Cas9. Nature 516:263-266

19. Kay MA (2011) State-of-the-art gene-based therapies: the road ahead. Nat Rev Genet:316-328.

20. Liu KC, Lin BS, Gao AD, Ma HY, Zhao M, et al. (2014) Integrase-Deficient Lentivirus: Opportunities and Challenges for Human Gene Therapy. Curr Gene Ther 14:352-364

21. Crystal RG (2014) Adenovirus: the first effective in vivo gene delivery vector. Hum Gene Ther 25:3-11.

22. Kotterman MA, Schaffer DV (2014) Engineering adeno-associated viruses for clinical gene therapy. Nat Rev Genet 15:445-451.

23. Selot RS, Hareendran S, Jayandharan GR (2014) Developing immunologically inert adeno-associated virus (AAV) vectors for gene therapy: possibilities and limitations. Curr Pharm Biotechnol 14:1072-1082.

24. Dismuke DJ, Tenenbaum L, Samulski RJ (2013) Biosafety of recombinant adeno-associated virus vectors. Curr Gene Ther 13:434-452.

25. Banasik MB, McCray PB Jr (2010) Integrase-defective lentiviral vectors: progress and applications. Gene Ther 17:150-157.

26. Cheng R, Peng J, Yan Y, Cao P, Wang J et al. (2014) Efficient gene editing in adult mouse livers via adenoviral delivery of CRISPR/Cas9. FEBS Lett588: 1

27. Ding Q, Strong A, Patel KM, Ng SL, Gosis BS, et al. (2014) Permanent alteration of PCSK9 with in vivo CRISPR-Cas9 genome editing. Circ Res 115:488-492.

28. Maggio I, Holkers M, Liu J, Janssen JM, Chen X, et al (2014) Adenoviral vector delivery of RNA-guided CRISPR/Cas9 nuclease complexes induces targeted mutagenesis in a diverse array of human cells.Sci Rep 4:5105.

29. Li M, Suzuki K, Kim NY, Liu GH, Izpisua Belmonte JC (2014) A cut above the rest: targeted genome editing technologies in human pluripotent stem cells. J BiolChem 289:4594-4599.

30. Senís E, Fatouros C, Große S, Wiedtke E, Niopek D, et al. (2014) CRISPR/ Cas9-mediated genome engineering: An adeno-associated viral vector. Biotechnol J 9:1402-1412.

31. Liu GH, Suzuki K, Qu J, Sancho-Martinez I, Yi F, Li M et al. (2011) Targeted gene correction of laminopathy-associated LMNA mutations in patient-specific iPSCs. Cell Stem Cell 8:688-694.

32. Nissan X, Blondel S, Navarro C, Maury Y, Denis C et al. (2012) Unique preservation of neural cells in Hutchinson- Gilford progeria syndrome is due to the expression of the neural-specific miR-9 microRNA. Cell Rep 2:1-9.

33. Ding DC, Shyu WC, Lin SZ (2011) Mesenchymal stem cells. Cell Transplant 20:5-14.

34. Ren G, Chen X, Dong F, Li W, Ren X et al. (2012) Concise review: mesenchymal stem cells and translational medicine emerging issues. Stem Cells Transl Med 1:51-58.

35. Dalberto TP, Nardi NB, Camassola M (2010) Mesenchymal stem cells as platform for gene therapy protocols. SciProg 93:129-140.

36. Lu ZH, Kaliberov S, Zhang J, Muz B, Azab AK, et al. (2014) The myeloidbinding peptide adenoviral vector enables multi-organ vascular endothelia gene targeting. Lab Invest 94:881-892.

37. Moreno E, Rhiner C (2014) Darwin's multicellularity: from neurotrophic theories and cell competition to fitness fingerprints. Curr Opin Cell Biol 31C:16-22.
Citation: Arancio W, Genovese SI, Pizzolanti G, Giordano C (2014) Hutchinson Gilford Progeria Syndrome: A Therapeutic Approach via Adenoviral Delivery of CRISPR/cas Genome Editing System. J Genet Syndr Gene Ther 6: 256. doi:10.4172/2157-7412.1000256
Submit your next manuscript and get advantages of OMICS Group submissions

\section{Unique features:}

- User friendly/feasible website-translation of your paper to 50 world's leading languages

Audio Version of published paper

- Digital articles to share and explore

Special features:

400 Open Access Journals

30,000 editorial team

21 days rapid review process

Quality and quick editorial, review and publication processing

Indexing at PubMed (partial), Scopus, EBSCO, Index Copernicus and Google Scholar et

Sharing Option: Social Networking Enabled

Authors, Reviewers and Editors rewarded with online Scientific Credits

Befter discount for your subsequent articles

Submit your manuscript at: http://www.editorialmanager.com/omicsgroup/ 